\title{
On the logical structure of choice and bar induction principles
}

\author{
Nuria Brede ${ }^{1}$ and Hugo Herbelin ${ }^{2}$ \\ ${ }^{1}$ University of Potsdam, Germany \\ ${ }^{2}$ Inria Paris, Université de Paris, CNRS, IRIF, France
}

\begin{abstract}
We develop an approach to choice principles and their contra-positive bar-induction principles as extensionality schemes connecting an "effective" or "intensional" view of respectively ill- and well-foundedness properties to an "extensional" or "ideal" view of these properties. After classifying and analysing the relations between different intensional definitions of ill-foundedness and well-foundedness, we introduce, for a domain $A$, a codomain $B$ and a "filter" $T$ on finite approximations of functions from $A$ to $B$, a generalised form GDC $A B T$ of the axiom of dependent choice and dually a generalised bar induction principle $\mathrm{GBI}_{A B T}$ such that:
\end{abstract}

$\mathrm{GDC}_{A B T}$ intuitionistically captures

- the strength of the general axiom of choice expressed as $\forall a \exists b R(a, b) \Rightarrow \exists \alpha \forall a R(a, \alpha(a)))$ when $T$ is a filter that derives point-wise from a relation $R$ on $A \times B$ without introducing further constraints,

- the strength of the Boolean Prime Filter Theorem / Ultrafilter Theorem if $B$ is the two-element set $\mathbb{B}$,

- the strength of the axiom of dependent choice if $A=\mathbb{N}$,

and up to weak classical reasoning

- the (choice) strength of Weak König's Lemma if $A=\mathbb{N}$ and $B=\mathbb{B}$.

$\mathrm{GBI}_{A B T}$ intuitionistically captures

- the strength of Gödel's completeness theorem in the form validity implies provability for entailment relations when $B=\mathbb{B}$,

- the strength of the Bar Induction when $A=\mathbb{N}$,

- the (choice) strength of the Weak Fan Theorem when $A=\mathbb{N}$ and $B=\mathbb{B}$.

Contrastingly, even though $\mathrm{GDC}_{A B T}$ and $\mathrm{GBI}_{A B T}$ smoothly capture several variants of choice and bar induction, some instances are inconsistent, e.g. when $A$ is $\mathbb{B}^{\mathbb{N}}$ and $B$ is $\mathbb{N}$.

\section{Introduction}

\subsection{Bar induction, dependent choice and their variants as extensionality principles}

For a domain $A$, there are different ways to define a well-founded tree branching over $A$. A first possibility is to define it as an inductive object built from leaves and from nodes associating a subtree to each element in $A$. We will call this definition intensional. Using a syntax familiar to functional programming languages or Martin-Löf-style type theory, such intensional trees correspond to inhabitants of an inductive type:

type tree $=$ Leaf $\mid$ Node of $(\mathrm{A} \rightarrow$ tree $)$

A second possibility is a definition which we shall call extensional and which is probably more standard in the context of non type-theoretic mathematics. Let $A^{*}$ denote the set of finite sequences of elements of $A$, with \langle\rangle denoting the empty sequence and $u \star a$ the extension of the sequence $u$ with $a$ from $A$. Then an extensional tree $T$ is a downwards-closed predicate over $A^{*}$. Finite sequences $u \in A^{*}$ are interpreted as finite paths from the root of a tree and the predicate determines which paths are contained in $T$. We say that $T$ is extensionally well-founded if for all infinite paths $\alpha$ in $A^{\mathbb{N}}$, the path eventually "leaves" the tree, i.e. there is an initial finite prefix $u$ of $\alpha$ such that $u$ (as path from the root) is not contained in $T$. 
The intensional definition is stronger: to any inductively-defined tree $t$, we can associate an extensionally well-founded tree $T(t)$ by recursion on $t$ as follows:

$$
\begin{array}{ll}
u \in T(\text { Leaf }) & \triangleq \perp \\
u \in T(\operatorname{Node}(f)) & \triangleq \top \\
u \star a \in T(\operatorname{Node}(f)) & \triangleq u \in T(f(a))
\end{array}
$$

and we can prove by induction on $t$ that $\forall \alpha \exists n T(t)\left(\alpha_{\mid n}\right)$, where $\alpha_{\mid n}$ is the restriction of $\alpha$ to its first $n$ values.

Bar induction, introduced by Brouwer and further analysed by Kleene and Vesley [25] is the converse property, namely that any extensionally well-founded $T$ can be turned into an inductively-defined tree. I.e. as a scheme it asserts the equivalence of the extensional and intensional definitions of well-foundedness ${ }^{1}$. This makes explicit the view developed in particular by Howard and Kreisel [15] that bar induction transforms extensional well-foundedness into well-founded induction (where the latter is seen as the ability to exhibit an explicit inductively-defined tree-like proof of being inductively barred).

To clarify what this means, and in particular to clarify in which case an inductively-defined $t$ denotes the same tree as an extensionally well-founded $T$ tree, we can define a realisability relation between $t$ and $T$ as follows:

- Leaf realises $T$ at $u$ if \langle\rangle$\notin T$

- Node $(f)$ realises $T$ at $u$ if for all $a, f(a)$ realises $T$ at $u \star a$

Then, we can prove by induction on $t$ that the subtree of $t$ rooted in $u$ realises $T(t)$ at $u$.

The notion inductively well-founded combines the data of a tree $t$ and the property that this tree $t$ realises a predicate: We define $T$ is inductively well-founded at $u$ as the smallest predicate which satisfies

- if $u \notin T$ then $T$ is inductively well-founded at $u$

- if, for all $a, T$ is inductively well-founded at $u \star a$, then $T$ is inductively well-founded at $u$

In particular, the existence of a tree $t$ such that $t$ realises $T$ at $u$ is equivalent to $T$ inductively well-founded at $u$ : the inductive structure of the subtree of $t$ rooted in $u$ is then reflected in the structure of the proof of $T$ inductively well-founded at $u$.

This allows to rephrase the correspondence between the existence of an inductively-defined tree $t$ and the existence of an extensionally well-founded tree $T$ into a correspondence talking in both cases of an arbitrary (a priori not well-founded) tree:

- if $T$ is inductively well-founded, it is extensionally well-founded (this is provable by induction on the proof of $T$ inductively well-founded)

- if $T$ is extensionally well-founded, it is inductively well-founded (this is a reformulation of bar induction using the more informative property of inductively well-founded - called inductively barred when it applies to the complement of the tree, as in the original formulation of bar induction - rather than just the existence of an inductively-defined tree)

Now, if bar induction is considered as an extensionality principle, the same should be the case for its contrapositive which is logically equivalent to the axiom of dependent choice: The axiom of dependent choice can be rephrased as a principle asserting that if a tree is coinductively ill-founded, then it is extensionally ill-founded (i.e. an infinite branch can be found). We will detail this in Section 2, together with precise relations between these principles and their restriction on finitely-branching trees, namely König's Lemma and the Fan Theorem, introducing a systematic terminology to characterise and compare these different variants.

Note that the approach to consider bar induction and choice principles as extensional principles is also consistent with the methodology developed e.g. by Coquand and Lombardi: to avoid the necessity of choice or bar-induction axioms, mathematical theorems are restated using the (co-)inductively-defined notions of well- and ill-foundedness rather than the extensional notions [9, 10].

\footnotetext{
${ }^{1}$ Kleene and Vesley [25] used respectively the terms "explicit" and "inductive".

${ }^{2}$ The spelling König's Lemma is also common. We respect here the original Hungarian spelling of the author.
} 
Table 1: Summary

\begin{tabular}{|c|c|}
\hline ill-founded-style & well-founded-style \\
\hline \multicolumn{2}{|c|}{ functions from $A$ to $B$} \\
\hline \multicolumn{2}{|l|}{$\mathrm{AC}_{A B R}=\mathrm{GDC}_{A B R_{\top}}$} \\
\hline \multicolumn{2}{|c|}{ branching from $\mathbb{N}$ over arbitrary $B$} \\
\hline $\mathrm{DC}_{B}^{\text {productive }}=\mathrm{GDC}_{\mathbb{N} B}$ & $\mathrm{BI}_{B}^{\text {ind }}=\mathrm{GBI}_{\mathbb{N} B}$ \\
\hline \multicolumn{2}{|c|}{ branching from $\mathbb{N}$ over non-empty finite $B$} \\
\hline $\mathrm{KL}=\mathrm{DC}_{\text {fin }}^{\text {staged }}$ & $\mathrm{FT}=\mathrm{Bl}_{\text {fin }}^{\text {uniform }}$ \\
\hline \multicolumn{2}{|c|}{ binary branching from $\mathbb{N}$} \\
\hline $\mathrm{WKL}=\mathrm{DC}_{\mathbb{B}}^{\text {staged }}$ & $\mathrm{WFT}=\mathrm{BI}_{\mathbb{B}}^{\text {uniform }}$ \\
\hline $\mathrm{WKL}={ }_{c o-\text { intuit. }} . \mathrm{GDC}_{\mathbb{N B}}$ & $\mathrm{WFT}={ }_{\text {intuitionistic }} \mathrm{GBI}_{\mathbb{N B}}$ \\
\hline \multicolumn{2}{|c|}{ binary branching from arbitrary $A$} \\
\hline $\begin{array}{l}\operatorname{Compl}_{A}^{-}(\mathcal{T})=\mathrm{GDC}_{A \mathbb{B} \mathcal{T} C} \\
\operatorname{BPF}_{\text {Free }(A)}\left(F_{\mathcal{T}}\right)=\mathrm{GDC}_{A \mathbb{B} \mathcal{T} C}\end{array}$ & $\operatorname{Compl}_{A}^{+}(\mathcal{T})=\mathrm{GBI}_{A \mathbb{B} \mathcal{T}}$ \\
\hline
\end{tabular}

\subsection{Weak König's Lemma at the intersection of Boolean Prime Filter Theorem and De- pendent Choice}

We know from reverse mathematics of the subsystems of second order arithmetic [24] that the binary form of König's lemma, namely Weak König's Lemma (WKL) has the strength of Gödel's completeness theorem (for a countable language). Reverse mathematics of the axiom of choice and its variants in set theory [13, 14, 18, 11] also tells that Gödel's completeness theorem has the strength of the Boolean Prime Filter Theorem (for a language of arbitrary cardinal). This suggests that the Boolean Prime Filter Theorem is the "natural" generalisation of WKL from countable to arbitrary cardinals.

On the other side, Weak Kônig's Lemma is a consequence ${ }^{3}$ of the axiom of Dependent Choice, the same way as its dual, the Weak Fan Theorem, is an instance of Bar Induction, itself classically equivalent to the axiom of Dependent Choice. This suggests that there is common principle which subsumes both the Axiom of Dependent Choices and the Boolean Prime Filter Theorem with Weak König's Lemma at their intersection.

Such a principle is stated in Section 3 where it is shown that the ill-founded version indeed generalises the axiom of Dependent Choice and the well-founded version generalises Bar Induction. In the same section, we also show that one of the instance of the ill-founded version captures the general Axiom of Choice, but that, in its full generality, the new principle is actually inconsistent.

Section 4 is devoted to show that the Boolean Prime Filter Theorem is an instance of the generalised axiom of Dependent Choice. In particular, this highlights that the notions of ideal and filter generalise the notion of a binary tree where the prefix order between paths of the tree is replaced by an inclusion order between non-sequentially ordered paths now seen as finite approximations of a function from $\mathbb{N}$ to the two-element set $\mathbb{B}$.

\subsection{Methodology and summary}

For our investigations to apply both to classical and to intuitionistic mathematics, we carefully distinguish between the choice axioms (seen as ill-foundedness extensionality schemes) and bar induction schemes (seen as well-foundedness extensionality schemes).

All in all, the classification we obtain is summarised in Table 1 where the definitions of the different notions can be found in the respective sections of the paper.

\footnotetext{
${ }^{3}$ Note that König's Lemma is a theorem of set theory and that we need to place ourselves in a sufficiently weak metatheory, e.g. RCA ${ }_{0}$, to state this result.
} 


\section{The logical structure of dependent choice and bar induction principles}

\subsection{Metatheory}

We place ourselves in a metatheory capable to express arithmetic statements. In addition to the type $\mathbb{N}$ of natural numbers together with induction and recursion, we assume the following constructions to be available:

- The type $\mathbb{B}$ of Boolean values 0 and 1 together with a mechanism of definition by case analysis. It shall be convenient to allow the definition of propositions by case analysis as in if $b$ then $P$ else $Q$, whose logical meaning shall be equivalent to $(b=1 \wedge P) \vee(b=0 \wedge Q)$.

- For any type $A$, the type $A^{*}$ of finite sequences over $A$ whose elements shall generally be ranged over by the letters $u, v \ldots$ We write \langle\rangle for the empty sequence and $u \star a$ for the extension of sequence $u$ with element $a$. We write $|u|$ for the length of $u$ and $u(n)$ for the $n^{\text {th }}$ element of $u$ when $n<|u|$. We write $v @ u$ for the concatenation of $v$ and $u$. We write $u \leq_{s} v$ to mean that $u$ is an initial prefix of $v$. This is inductively defined by:

$$
\overline{u \leq_{s} u} \quad \frac{u \leq_{s} v}{u \leq_{s} v \star a}
$$

We shall also support case analysis over finite sequences under the form of a case operator.

- For any two types $A$ and $B$, the type $A \rightarrow B$ of functions from $A$ to $B$. Functions can be built by $\lambda$-abstraction as in $\lambda x . t$ for $x$ in $A$ and $t$ in $B$ and used by application as in $t(u)$ for $t$ in $A \rightarrow B$ and $u$ in $A$. To get closer to the traditional notations, we shall also abbreviate $t\left(u_{1}\right) \ldots\left(u_{n}\right)$ into $t\left(u_{1}, \ldots, u_{n}\right)$.

- A type Prop reifying the propositions as a type. The type $A \rightarrow$ Prop shall then represent the type of predicates over $A$. We shall allow predicates to be defined inductively (smallest fixpoint) or coinductively (greatest fixpoint), using respectively the $\mu$ and $\nu$ notations.

- For any type $A$ and predicate $P$ over $A$, the subset $\{a: A \mid P(a)\}$ of elements of $A$ satisfying $P$.

This is a language for higher-order arithmetic but in practice, we shall need quantification just over functions and predicates superficially of rank 1 (i.e. of the form $A_{1} \rightarrow \ldots \rightarrow A_{n} \rightarrow A$ or $A_{1} \rightarrow \ldots \rightarrow A_{n} \rightarrow$ Prop with no arrow types in $A$ and the $A_{i}$ ). We however also allow arbitrary type constants to occur, so we can think of our effective metatheory as a second-order arithmetic generic over arbitrary more complex types. In practise, our metatheory could typically be the image of arithmetic in set theory or in an impredicative type theory. We will in any case use the notation $a \in A$ to mean that $a$ has type $A$ when $A$ is a type, which, if in set theory, will become $a$ belongs to the set $A$.

We shall leave open the meaning of the connectives. Using linear logic as a reference for the semantics of connectives, we shall interpret implication either linearly (i.e. as linear logic's $P \multimap Q$ where $P$ can be used only once in a proof of $Q$ ), intuitionistically (i.e. as $! P \multimap Q$ where ! is the of-course modality of linear logic expressing that $P$ can be used an arbitrary number of times in the proof), or classically (i.e. as $! P \multimap ? Q$ where ? is the why-not modality of linear logic, expressing that classical reasoning can be used to prove $Q)$. Similarly, $\exists a^{A} Q$ shall have an intuitionistic/linear meaning (i.e. as $\bigoplus_{a \in A} Q$ using notations of linear logic) or a classical meaning (i.e. as ? $\bigoplus_{a \in A} Q$ ).

\subsection{Infinite sequences}

We write $A^{\mathbb{N}}$ for the infinite (countable) sequences of elements of $A$. There are different ways to represent such an infinite sequence:

- We can represent it as a function, i.e. as a functional object of type $\mathbb{N} \rightarrow A$.

- We can represent it as a total functional relation, i.e. as a relation $R$ of type $\mathbb{N} \rightarrow A \rightarrow$ Prop such that $\forall n \exists ! a R(n, a)$.

- Additionally, when $A$ is $\mathbb{B}$, an extra possible representation shall be as predicate $P$ over $\mathbb{N}$ with intended meaning 1 if $P(n)$ holds and 0 if $\neg P(n)$ holds (and unknown meaning otherwise).

The representation as a functional relation is weaker in the sense that a function $\alpha$ mechanically induces a functional relation $\lambda n . \lambda a . \alpha(n)=a$ but the converse requires the axiom of unique choice. In the sequel, we shall use the notation $\alpha(n)=a$ and $\alpha(n) \triangleq a$ to mean different things depending on the representation chosen for $\mathbb{N} \rightarrow B$. 
Table 2: Logically equivalent dual concepts on dual predicates

\begin{tabular}{|c|c|}
\hline$T$ is a tree & $T$ is monotone \\
(closure under restriction) & (closure under extension) \\
$\forall u \forall a(u \star a \in T \Rightarrow u \in T)$ & $\forall u \forall a(u \in T \Rightarrow u \star a \in T)$ \\
\hline
\end{tabular}

In the first case, $\alpha(n)=a$ shall mean $\alpha(n)=a$ where the equality sign of the notation is interpreted as the equality on $A$. Similarly, $\alpha(n) \triangleq a$ shall define the function $\alpha \triangleq \lambda n$. $a$.

In the second case, $\alpha(n)=a$ shall however mean $\alpha(n, a)$ and $\alpha(n) \triangleq a$ shall define the functional relation $\alpha \triangleq$ $\lambda\left(n, a^{\prime}\right) . a^{\prime}=a$ where $n$ can occur in $a$.

When $A$ is $\mathbb{B}$, the representation as a predicate $P$ is even weaker in the sense that a functional relation $R$ induces a predicate $\lambda n . R(n, 1)$ but the converse requires classical reasoning. We can easily turn a predicate $P$ into a relation $\lambda n$. $\lambda b$.if $b$ then $P(n)$ else $\neg P(n)$ but proving $\forall n \exists ! b$ if $b$ then $P(n)$ else $\neg P(n)$ requires a call to excluded-middle on $P(n)$.

When $A$ is $\mathbb{B}$ and $\alpha$ is a predicate, we shall define $\alpha(n)=1$ as $\alpha(n)$ and $\alpha(n)=0$ as $\neg \alpha(n)$. Technically, this means seeing $\alpha(n)=b$ as a notation for if $b$ then $\alpha(n)$ else $\neg \alpha(n)$. Similarly, $\alpha(n) \triangleq b$ shall define $\alpha \triangleq \lambda n$. if $b$ then $T$ else $\perp$.

In particular, this means that all choice and bar induction statements of this paper have two readings of a different logical strength (depending on the validity of the axiom of unique choice in the metatheory), or even three readings (depending on the validity of the axiom of unique choice and of classical reasoning) when the codomain of the function mentioned in the theorems is $\mathbb{B}$.

If $\alpha \in A^{\mathbb{N}}$, we write $u \prec_{s} \alpha$ to mean that $u$ is an initial prefix of $f$. This shall be defined inductively by the following clauses:

$$
\overline{\langle\rangle \prec_{s} \alpha} \quad \frac{u \prec_{s} \alpha \quad \alpha(|u|)=a}{u \star a \prec_{s} \alpha}
$$

If $a \in A$ and $\alpha \in A^{\mathbb{N}}$, we write $a @ \alpha$ for the sequence $\beta$ defined by $\beta(0) \triangleq a$ and $\beta(n+1) \triangleq \alpha(n)$.

We have the following easy property:

Proposition 1 If $u \prec_{s} \alpha$ then $a @ u \prec_{s} a @ \alpha$.

\subsection{Trees and monotone predicates}

Let $B$ be a type and $T$ be a predicate on $B^{*}$. We overload the notation $u \in T$ to mean that $T$ holds on $u \in B^{*}$. We say that $T$ is finitely-branching if $B$ is in bijection with a non-empty bounded subset of $\mathbb{N}$ (i.e. to $\{n: \mathbb{N} \mid n \leq p\}$ for some $p$ ).

We say that $T$ is a tree if it is closed under restriction, and, dually, that $T$ is monotone if it is closed under extension (the formal definitions are given in Table 2). Classically, we have $T$ monotone iff $\neg T$ is a tree, and, dually, $\neg T$ monotone iff $T$ is a tree. In particular, another way to describe a tree is as an antimonotone predicate ${ }^{4}$. It is convenient for the underlying intuition to restrict oneself to predicates which are trees, or which are monotone, even if it does not always matter in practice. When it matters, a predicate is turned into a tree either by discarding sequences not connected to the root or by completing it with missing sequences from the root: these are respectively the downwards arborification $\downarrow^{-} T$ and upwards arborification $\uparrow^{-} T$ of a predicate, as shown in Table 3 . We dually write $\uparrow^{+} T$ and $\downarrow^{+} T$ for the upwards monotonisation and downwards monotonisation of $T$. Arborification and monotonisation are idempotent. We shall in general look for minimal definitions of the concept involved in the paper, and thus consider arbitrary predicates as much as possible, turning them into trees or monotone predicates only when needed to give sense to the definitions.

\subsection{Well-foundedness and ill-foundedness properties}

We list properties on predicates which are relevant for stating ill-foundedness axioms (i.e. choice axioms), and their dual well-foundedness axioms (i.e. bar induction axioms). Duality can be understood both under a classical or linear interpretation of the connectives, where the predicate $T$ in one column is supposed to be dual of the predicate $T$ occurring in the other column (dual predicates if in linear logic, negated predicates if in classical logic). Table 4 details properties which differ by contraposition and are thus logically equivalent (in classical and linear logic). On the other side, tables 5 and 6 detail properties which are logically opposite.

\footnotetext{
${ }^{4}$ From a categorical perspective, a tree is a contravariantly functorial predicate over the preorder generated by $u \leq_{s} v$, while a monotone predicate is covariantly functorial.
} 
Table 3: Logically opposite arborification/monotonisation on dual predicates

\begin{tabular}{|c|c|}
\hline $\begin{array}{l}\text { downwards arborification } \\
\text { of } T \\
\qquad\left(\downarrow^{-} T\right)\end{array}$ & $\begin{array}{l}\text { upwards monotonisation } \\
\text { of } T \\
\qquad\left(\uparrow^{+} T\right)\end{array}$ \\
\hline$\lambda u . \forall u^{\prime}\left(u^{\prime} \leq_{s} u \Rightarrow u^{\prime} \in T\right)$ & $\lambda u . \exists u^{\prime}\left(u^{\prime} \leq_{s} u \wedge u^{\prime} \in T\right)$ \\
\hline $\begin{array}{l}\text { upwards arborification of } \\
\qquad(\uparrow-T)\end{array}$ & $\begin{array}{c}\text { downwards } \\
\text { monotonisation of } T \\
\left(\downarrow^{+} T\right)\end{array}$ \\
\hline$\lambda u . \exists u^{\prime}\left(u \leq_{s} u^{\prime} \wedge u^{\prime} \in T\right)$ & $\lambda u . \forall u^{\prime}\left(u \leq_{s} u^{\prime} \Rightarrow u^{\prime} \in T\right)$ \\
\hline
\end{tabular}

Table 4: Basic logically equivalent dual properties on dual predicates

\begin{tabular}{|c|c|}
\hline$T$ is progressing at $u\left(^{*}\right)$ & $T$ is hereditary at $u$ \\
$u \in T \Rightarrow(\exists a u \star a \in T)$ & $(\forall a u \star a \in T) \Rightarrow u \in T$ \\
\hline$T$ is progressing $\left(^{*}\right)$ & $T$ is hereditary \\
$\forall u(T$ is progressing at $u)$ & $\forall u(T$ is hereditary at $u)$ \\
\hline
\end{tabular}

We indicated with $\left(^{*}\right)$ concepts for which we did not find an existing terminology in the literature. Thus, the terminology is ours. Also, what we called staged infinite is often simply called infinite. We used staged infinite to make explicit the difference from a definition based on the presence of an infinite number of nodes. Thereby we also obtain a symmetry with the notion of staged barred. What we call having an infinite branch could alternatively be called ill-founded, or having a choice function. In particular, the terminology having an infinite branch applies here to any predicate and is not restricted to trees. Note that well-founded in the standard meaning is the same as barred for the dual predicate. In particular, when opposing ill-foundedness and well-foundedness, we adopt a bias towards the tree view, i.e. towards the left column. We have the following:

Proposition 2 If $T$ is a tree, then having unbounded paths is equivalent to being staged infinite. Dually, if $T$ is monotone, being a uniform bar is equivalent to being staged barred.

Proof: Because trees and monotone predicates are invariant under arborification and monotonisation.

As a consequence, it is common to use the notion of staged infinite, which is simpler to formulate, when we know that $T$ is a tree. Otherwise, if $T$ is an arbitrary predicate which is not necessarily a tree, there is no particular interest in using the notion of staged infinite. Similarly, staged barred is a simpler way to state uniformly barred when $T$ is monotone, i.e., conversely, uniform bar is the expected refinement of staged barred when $T$ is not known to be monotone.

By a direct coinduction, any spread is productive, and further, any spread is productive at any $u \in T$. Thus, we have:

Proposition $3 T$ spread implies $T$ productive, and, dually, $T$ inductively barred implies $T$ resisting.

Conversely, a productive $T$ may be productive at \langle\rangle without being productive at all $u \in T$, so we may need to prune $T$ to extract from it a spread. Dually, not all resisting predicates are inductive bars but we can saturate them into inductive bars, using the notion of adherence. We make this formal in the following proposition:

Proposition 4 If $T$ is productive then its pruning is a spread. Dually, if $T$ resists then its adherence is an inductive bar.

Proof: That \langle\rangle is in the pruning of $T$ is direct from $T$ productive. That the pruning of $T$ is progressing on all $u$ is also direct by construction of the pruning. The other part of the statement is by duality.

Also, we can relate productive and spread, as well as inductive bar and resisting as follows:

Proposition $5 T$ is productive iff there exists $U \subset T$ which is a spread. Dually, $T$ is an inductive bar iff all $U \supset T$ resists. 
Table 5: Logically opposite dual properties on dual predicates

\begin{tabular}{|c|c|}
\hline ill-foundedness properties & well-foundedness properties \\
\hline \multicolumn{2}{|c|}{ Intensional concepts } \\
\hline$T$ is a spread & $T$ is resisting $(*)$ \\
\hline\langle\rangle$\in T \wedge T$ progressing & $T$ hereditary $\Rightarrow\langle\rangle \in T$ \\
\hline pruning of $T$ & adherence $(*)$ of $T$ \\
\hline$\nu X . \lambda u .(u \in T \wedge \exists a u \star a \in X)$ & $\mu X . \lambda u .(u \in T \vee \forall a u \star a \in X)$ \\
\hline$T$ is productive & $T$ is inductively barred \\
\hline\langle\rangle$\in$ pruning of $T$ & \langle\rangle$\in$ adherence of $T$ \\
\hline \multicolumn{2}{|c|}{ Intensional concepts relevant for the finite case } \\
\hline$T$ has unbounded paths & $T$ is uniformly barred \\
\hline$\forall n \exists u\left(|u|=n \wedge u \in \downarrow^{-} T\right)$ & $\exists n \forall u\left(|u|=n \Rightarrow u \in \uparrow^{+} T\right)$ \\
\hline$T$ is staged infinite & $T$ is staged barred $(*)$ \\
\hline$\forall n \exists u(|u|=n \wedge u \in T)$ & $\exists n \forall u(|u|=n \Rightarrow u \in T)$ \\
\hline \multicolumn{2}{|c|}{ Extensional concepts } \\
\hline$T$ has an infinite branch & $T$ is barred \\
\hline$\exists \alpha \forall u\left(u \prec_{s} \alpha \Rightarrow u \in T\right)$ & $\forall \alpha \exists u\left(u \prec_{s} \alpha \wedge u \in T\right)$ \\
\hline
\end{tabular}

Table 6: Useful relativisation of some of the concepts of Table 5

\begin{tabular}{|c|c|}
\hline ill-foundedness-style & well-foundedness-style \\
\hline \multicolumn{2}{|c|}{ Relativised intensional concepts } \\
\hline$T$ is productive from $u$ & $T$ is inductively barred from $u$ \\
$u \in$ pruning of $T$ & $u \in$ adherence of $T$ \\
\hline Relativised intensional concepts relevant for the finite case \\
\hline$T$ has unbounded paths from $u$ & $T$ is uniformly barred from $u$ \\
$\forall n \exists u^{\prime}\left(\left|u^{\prime}\right|=n \wedge u @ u^{\prime} \in \downarrow^{-} T\right)$ & $\exists n \forall u^{\prime}\left(\left|u^{\prime}\right|=n \Rightarrow u @ u^{\prime} \in \uparrow^{+} T\right)$ \\
\hline$T$ is staged infinite from $u$ & $T$ is staged barred from $u^{\prime}$ \\
$\forall n \exists u^{\prime}\left(\left|u^{\prime}\right|=n \wedge u @ u^{\prime} \in T\right)$ & $\exists n \forall u^{\prime}\left(\left|u^{\prime}\right|=n \Rightarrow u @ u^{\prime} \in T\right)$ \\
\hline \multicolumn{2}{|c|}{ Extensional concepts } \\
\hline$T$ has an infinite branch from $u$ & $T$ is barred from $u$ \\
$\exists \alpha \forall u^{\prime}\left(u^{\prime} \prec_{s} \alpha \Rightarrow u @ u^{\prime} \in T\right)$ & $\forall \alpha \exists u^{\prime}\left(u^{\prime} \prec_{s} \alpha \wedge u @ u^{\prime} \in T\right)$ \\
\hline
\end{tabular}


Proof: By duality, it is enough to prove the first equivalence. From left to right, we use Proposition 4, observing that the pruning of $T$ is included in $T$. From right to left, a spread is productive and a coinduction suffices to prove that inclusion preserves productivity.

On the other side, having unbounded paths is equivalent to being a spread or to being productive only when $T$ is finitely-branching. Similarly for being uniformly barred compared to being an inductive bar or being resisting. Moreover, none of the equivalences hold linearly. The second one requires intuitionistic logic, i.e. requires the ability to use an hypothesis several times while the first one, dually, requires a bit of classical reasoning ${ }^{5}$.

For $S$ being a class of formulae and $P$ and $Q$ ranging over $S$, let $D_{S}$ be the principle $\left.\forall x y(P(x) \vee Q(y))\right) \Rightarrow(\forall x P(x)) \vee$ $(\forall y Q(y))$. Dually, let $C_{S}$ be $(\exists x P(x)) \wedge(\exists y Q(y)) \Rightarrow \exists x \exists y(P(x) \wedge Q(y))$.

Proposition 6 If $B$ is non-empty finite, then productive is equivalent to having unbounded paths and being an inductive bar is equivalent to uniformly barred. The first statement holds in a logic where $D_{S}$ holds and the second in a logic where $C_{S}$ holds, for $S$ a class of formulae containing arithmetical existential quantification over $T$.

Proof: Relying on duality, we only prove the first statement. Without loss of generality, we also assume that $B$ is $\mathbb{B}$. Our proof relies on an argument found in $[3,16]$ and proceeds by proving more generally that $T$ is productive from $u$ iff $T$ has unbounded paths from $u$.

From left to right, we reason by induction on $n$. If $n$ is 0 this is direct from $T$ productive by defining $u^{\prime} \triangleq\langle\rangle$. Otherwise, by $T$ productive from $u$, we get $a$ such that $T$ is productive from $u \star a$, obtaining by induction $u^{\prime}$ of length $n-1$ such that $(u \star a) @ u^{\prime}=u @\left(a @ u^{\prime}\right) \in \downarrow^{-} T$, showing that $a @ u^{\prime}$ is the expected sequence of length $n$.

From right to left, we reason coinductively. To prove that $u \in T$, we take a path of length 0 . Then, in order to apply the coinduction hypothesis and prove the coinductive part, we prove that there is $b$ such that $T$ has unbounded paths from $u \star b$. By $D_{S}$, it is enough to prove that for all $n_{0}$ and $n_{1}$, there is a path $u_{0}$ of length $n_{0}$ and a path $u_{1}$ of length $n_{1}$ such that either $(u \star 0) @ u_{0}$ or $(u \star 1) @ u_{1}$ is in $\downarrow^{-} T$. So, let $n_{0}$ and $n_{1}$ be given lengths. By unbounded paths from $u$, we get a sequence $u^{\prime \prime}$ of length $\max \left(n_{0}, n_{1}\right)+1$ such that $u @ u^{\prime \prime} \in \downarrow^{-} T$. This is a non-empty sequence, hence a sequence of the form $b @ u^{\prime}$ so that we have either $(u \star 0) @ u^{\prime} \in \downarrow^{-} T$ or $(u \star 1) @ u^{\prime} \in \downarrow^{-} T$ for $u^{\prime}$ of length $\max \left(n_{0}, n_{1}\right)$. By closure of $\downarrow^{-} T$, prefixes $u_{0}$ of length $n_{0}$ and $u_{1}$ of length $n_{1}$ of $u^{\prime}$ can be extracted which both are in $\downarrow^{-} T$.

Remark: Based on the decomposition of WKL for decidable trees into a choice principle and the Lesser Limited Principle of Omniscience (LLPO), we suspect that we actually have the stronger result that the equivalence of unbounded paths and productivity implies $D_{S}$ for the corresponding underlying class of formulae $S$, and similarly with $C_{S}$ and the dual statement.

\subsection{Standard bar induction and dependent choice principles}

In Table 7, we reformulate using our definitions standard statements of bar induction and dependent choice axioms from the literature. What we call Generic Bar Induction, shortly BI ind, is a rather standard form [25], apart from the fact that we do not fix in advance the logical complexity of $B$ - such as being countable or not - or the arithmetic strength of $T$ i.e. whether it is decidable, or recursively enumerable, etc. For dependent choice ${ }^{6}$, we consider here a pruned-tree-based definition $D C^{\text {spread }}$ corresponding to the instance $D_{\aleph_{\aleph_{0}}}$ of Levy's family of Dependent Choice indexed on cardinals [19] ${ }^{7}$. A comparison with other logically equivalent definitions of dependent choice will be given in Section 2.7 .

The Fan Theorem is sometimes stated over finitely-branching trees, where the definition of finite itself may vary [25, 16], but it is also sometimes considered by default to be on a binary tree $[4,2,3,7,9,17]$ in which case the finite version is sometimes called extended. What we call here Fan Theorem is a definition where the finite branching is uniform at all levels and we shall later call the binary version Weak Fan Theorem in Section 4. The statement of the Fan Theorem sometimes relies on the notion of inductive bar (e.g. [9]), or on the definition of staged barred for monotone predicates (as a variant in [17]), or on the dual notions of finite tree (i.e., technically of staged barred for the negation of a tree) and well-founded tree (i.e., technically of inductively barred for the negation of a tree) in e.g. [5]. But it also often relies on the definition of uniform bar [2, 3, 4, 7, 16, 17, 25] over an arbitrary predicate. It is this last variant which here we will explicitly call Fan Theorem. Note that as in the case of bar induction, we omit the usual restriction of the statement of the Fan Theorem to decidable predicates.

König's Lemma is generally stated as $T$ infinite implies $T$ has an infinite branch, but the definition of $T$ infinite may differ from author to author. We here use the name König's Lemma for the definition from [5, 16] which expresses

\footnotetext{
${ }^{5}$ or, to be more precise, co-intuitionistic reasoning, that is, using a multi-conclusion sequent calculus to formulate the reasoning, with the contraction rule allowed on conclusions but not on hypotheses

${ }^{6}$ or dependent choices for some authors, e.g. [18]

${ }^{7}$ Alternatively, it can be seen as the generalisation to arbitrary codomains of the Boolean dependent choice principle $D C{ }^{\vee}$ described e.g. in Ishihara [16].
} 
Table 7: Tree-based choice principles and bar induction principles

\begin{tabular}{|c|c|}
\hline ill-foundedness-style & well-foundedness-style \\
\hline $\begin{array}{l}\text { Tree-based Dependent Choice (DC }{ }^{\text {spread }} \text { ) } \\
T \text { spread } \Rightarrow T \text { has an infinite branch }\end{array}$ & $\begin{array}{l}\text { Dual to Tree-based Dep. Choice }\left(\mathrm{Bl}^{\text {resists }}\right) \\
T \text { barred } \Rightarrow T \text { resists }\end{array}$ \\
\hline $\begin{array}{l}\text { Dual to Generic Bar Induction (DCproductive) } \\
T \text { productive } \Rightarrow T \text { has an infinite branch }\end{array}$ & $\begin{array}{l}\text { Generic Bar Induction }\left(\left.\mathrm{B}\right|^{\text {ind }}\right) \\
T \text { barred } \Rightarrow T \text { inductively barred }\end{array}$ \\
\hline $\begin{array}{l}\text { Dual to Fan Theorem }\left(\mathrm{DC}_{\text {fin }}^{\text {unbounded }}\right) \\
T \text { finitely-branch. w. unbounded paths } \Rightarrow T \text { has an } \\
\text { infinite branch }\end{array}$ & $\begin{array}{l}\text { Fan Theorem }\left(\mathrm{Bl}_{\text {fin }}^{\text {uniform }}=\mathrm{FT}\right) \\
T \text { barred and finitely-branching } \Rightarrow T \text { uniform bar }\end{array}$ \\
\hline $\begin{array}{l}\text { König's Lemma }\left(\mathrm{DC}_{\text {fin }}^{\text {staged }}=\mathrm{KL}\right) \\
T \text { finitely-branching staged-infinite tree } \Rightarrow T \text { has an } \\
\text { infinite branch }\end{array}$ & $\begin{array}{l}\text { Dual to Kônig's Lemma }\left(\mathrm{B}_{\text {fin }}^{\text {staged }}\right) \\
T \text { barred, finitely-branching and monotone } \Rightarrow T \\
\text { staged barred }\end{array}$ \\
\hline
\end{tabular}

explicitly that the infinity can only be in depth. It does so by requiring arbitrary long branches rather than an infinite number of nodes. The exact definition of arbitrarily long branches also depends on authors. For instance, [26] relies (up to classical reasoning) on having unbounded paths for arbitrary predicates, but most of the time it is about what we call staged infinite tree $[3,16,17]$.

Note that there is a huge body of literature on bar induction, dependent choice, König's Lemma and the Fan Theorem, especially in the context of constructive mathematics. We apologise for not being exhaustive.

Otherwise said, the Fan Theorem and König's Lemma are dual up to the (small and somewhat insignificant) difference that in the literature we found König's Lemma mostly formulated on trees while the Fan Theorem was mostly formulated for arbitrary predicates. In particular, there is a standard way to go from arbitrary predicates to trees or monotone predicates by associating to each predicate its tree or monotone closure. Using the closure under restriction and closure under extension of a predicate, we can connect König's Lemma to the dual of the Fan Theorem and the Fan Theorem to the dual of König's Lemma.

Proposition 7 As schemes, that is when generalised over trees, it is equivalent to state König's Lemma on trees using staged-infinity ( $D C_{\mathrm{fin}}^{\text {staged}}$ ) or on arbitrary predicates using has unbounded paths ( $\left.D C_{\mathrm{fin}}^{\text {unbounded }}\right)$. Similarly, it is equivalent to state the Fan Theorem on monotone predicates using staged barred ( $B$ fin figed $^{\text {tag }}$ ) or on arbitrary predicates using uniformly barred (B/ finiform).

Proof: We treat the first equivalence. From left to right, if $T$ is a predicate, we apply $D C_{\text {fin }}^{\text {staged }}$ to $\downarrow^{-} T$. The resulting infinite branch is an infinite branch in $T$ because $\downarrow^{-} T \subset T$. From right to left, the statement holds by Proposition 2 . The second equivalence is by duality.

Similarly, Tree-based Dependent Choice and Bar Induction are dual except that Tree-based Dependent Choice is mostly stated for spreads while Bar Induction is stated for (the dual of) productive. Again, there are standard ways to pass from spread or co-spread to productive trees or inductive bars, and from a statement on serial or monotone relations to a statement on productive or inductively barred trees.

Proposition 8 As schemes, $D C^{\text {spread }}$ and $D C^{\text {productive }}$ are equivalent, and so are $B F^{\text {resists }}$ and $B F^{\text {ind }}$.

Proof: By Proposition 5.

\subsection{Choice and bar induction as relating intensional and extensional concepts}

The intensional definitions are stronger than the extensional ones, which implies that the choice and bar induction axioms can alternatively be seen as stating the logical equivalence of the intensional and extensional versions of ill-foundedness and well-foundedness properties (of various strengths).

Theorem $1 T$ inductively barred implies $T$ barred. Dually, $T$ has an infinite branch implies $T$ is productive. 
Proof: We prove by induction on the definition of $T$ inductively barred that $T$ inductively barred at $u$ implies $T$ barred from $u$ where the latter requires that for all $\alpha$, there is $v \prec_{s} \alpha$ such that $u v \in T$.

If $u \in T$, then it is enough to take \langle\rangle for $v$ to get $u @\langle\rangle \in T$ for any $\alpha$. If $T$ is barred from $u \star b \in T$ for all $b \in B$, this means that there is $v \prec_{s} \beta$ such that $(u \star b) @ v \in T$ for any $\beta$. For a given $\alpha$, set $b \triangleq \alpha(0)$ and $\beta(n) \triangleq \alpha(n+1)$ so that we can find $v \prec_{s} \beta$, hence $b @ v \prec_{s} b @ \beta$, i.e. $b @ v \prec_{s} \alpha$ (by Prop. 1) together with $u @(b @ v) \in T$.

The dual proof builds $T$ productive at $u$ from $T$ has an infinite branch from $u$ by coinduction. From the infinite branch $\alpha$ from $u$ and \langle\rangle$\prec_{s} \alpha$ we get $u @\langle\rangle \in T$, i.e. $u \in T$. It remains to find $b$ such that $T$ is productive from $u \star b$ and it suffices to take $\alpha(0)$ since $T$ has an infinite branch $\beta(n) \triangleq \alpha(n+1)$ from $u \star \alpha(0)$ simply because $v \prec_{s} \alpha$ implies $\alpha(0) @ v \prec_{s} \alpha(0) \star \beta$ (by Prop. 1) and $(u \star \alpha(0)) @ v \in T$ from $u @(\alpha(0) @ v) \in T$.

\subsection{Relation to other formulations of Dependent Choice and to countable Zorn's Lemma}

For $R$ a relation on $B$, it is common to formulate dependent choice as $\forall b^{B} \exists b^{B} R\left(b, b^{\prime}\right) \Rightarrow \forall b_{0}{ }^{B} \exists f^{\mathbb{N} \rightarrow B}\left(f(0)=b_{0} \wedge\right.$ $\forall n R(f(n), f(n+1)))$. Let us call serial a (homogeneous) relation such that $\forall b^{B} \exists b^{\prime}{ }^{B} R\left(b, b^{\prime}\right)$ holds. In this section, we formally compare the resulting statement of dependent choice to $D C_{B T}^{\text {productive }}$, examining also dual statements.

Let $R$ be a serial relation, i.e. a relation such that $\forall b^{B} \exists b^{\prime}{ }^{B} R\left(b, b^{\prime}\right)$. Using a seed $b_{0}$, each such relation $R$ can be turned into a predicate on $B^{*}$ under the two following ways:

- The chaining $R_{\top}^{*}\left(b_{0}\right)$ from $b_{0}$ is probably the most natural one: it says that $u \in R_{\top}^{*}\left(b_{0}\right)$ if all steps in $u$ from $b_{0}$ are in $R$.

- The alignment $R_{\uparrow}^{\triangleright}\left(b_{0}\right)$ from $b_{0}$ artificially uses non-empty sequences to represent pairs of elements. We have $u \in$ $R_{\top}^{\triangleright}\left(b_{0}\right)$ either when $u$ has at least two elements and the last two elements are related by $R$, or, when the sequence contains exactly one element which is related to $b_{0}$, or, finally, when the sequence is simply empty.

Reasoning by induction on $v \leq_{s} u$ in one direction and on $u$ in the other direction, we can show that both are related:

Proposition $9 u \in R_{\top}^{*}\left(b_{0}\right)$ iff $u \in \downarrow^{-} R_{\top}^{\triangleright}\left(b_{0}\right)$

Dually, we can define antichaining and blockings such that:

Proposition $10 u \in R_{\perp}^{*}\left(b_{0}\right)$ iff $u \in \uparrow^{+} R_{\perp}^{\triangleright}\left(b_{0}\right)$

The formal definitions are given in Table 8, where we can notice that the use of $\mu$ vs. $\nu$ does not matter in practice since the structure of the relation is a function of $|u|$.

We are now in position to state in Table 9 a relatively standard form of Dependent Choice which we call $\mathrm{DC}_{B R b_{0}}^{\text {serial }}$ for $R$ being a relation on $B$ and $b_{0}$ a seed in $B$. Though to our knowledge uncommon in the literature, we also mention its dual which we call $\mathrm{Bl}_{B R b_{0}}^{\text {least }}$.

We state a few results that allow to show the equivalence of $D C_{B R b_{0}}^{\text {serial }}$ and $D C_{B T}^{\text {productive }}$ as schemes.

We have the following properties.

Proposition $11 R$ serial implies $R_{\top}^{\triangleright}\left(b_{0}\right)$ productive for any $b_{0}$. Dually, if $R_{\perp}^{\triangleright}\left(b_{0}\right)$ is inductively barred then $R$ has a least element.

Proof: We prove by coinduction that $u \in R_{\top}^{\triangleright}\left(b_{0}\right)$ implies $R_{\top}^{\triangleright}\left(b_{0}\right)$ productive from $u$. If $u$ is empty, $R_{\top}^{\triangleright}\left(b_{0}\right)(\langle\rangle)$ holds by definition and there is by seriality a $b_{1}$ such that $R_{\top}^{\triangleright}\left(b_{0}\right)\left(b_{1}\right)$. This allows to conclude by coinduction hypothesis. If $u$ has the form $u^{\prime} \star b$, there is also by seriality a $b^{\prime}$ such that $R_{\top}^{\triangleright}\left(b_{0}\right)\left(u^{\prime} \star b \star b^{\prime}\right)$ and we can again conclude by coinduction hypothesis. The productivity of $R_{\top}^{\triangleright}\left(b_{0}\right)$ finally follows because $R_{\uparrow}^{\triangleright}\left(b_{0}\right)(\langle\rangle)$ holds by definition. The dual statement is by dual (inductive) reasoning.

Conversely, for $T$ a predicate, let $B_{T}$ be defined by $B_{T} \triangleq\left\{u \in B^{*} \mid T\right.$ is productive from $\left.u\right\}$ and let $R_{T}$ be the relation on $B_{T}$ defined by $R_{T}\left(u, u^{\prime}\right) \triangleq \exists b\left(u \star b=u^{\prime}\right)$. The relation $R_{T}$ is serial by construction: for $u$ such that $T$ is productive from $u$, there is $a$ such that $T$ is productive from $u \star a$ and $u \star a \in T$. Also, \langle\rangle$\in B_{T}$ as soon as $T$ is productive.

We can now formally state the correspondence in our language:

Theorem 2 As schemes, $D C_{B T}^{\text {productive }}$ and $D C_{B R b_{0}}^{\text {serial }}$ are logically equivalent. 
Table 8: Logically opposite dual concepts on dual homogeneous relations

\begin{tabular}{|c|c|}
\hline ill-foundedness-style & well-foundedness-style \\
\hline \multicolumn{2}{|c|}{ Intensional concepts } \\
\hline$R$ serial & $R$ has a "least" element \\
\hline$\forall b \exists b^{\prime} R\left(b, b^{\prime}\right)$ & $\exists b \forall b^{\prime} R\left(b, b^{\prime}\right)$ \\
\hline$R$ left-not-full $(*)$ & $R$ has a "maximal" element \\
\hline$\forall b \exists b^{\prime} \neg R\left(b, b^{\prime}\right)$ & $\exists b \forall b^{\prime} \neg R\left(b, b^{\prime}\right)$ \\
\hline chaining of $R$ from $b_{0}\left(R_{\top}^{*}\left(b_{0}\right)\right)$ & antichaining of $R$ from $b_{0}\left(R_{\perp}^{*}\left(b_{0}\right)\right)$ \\
\hline$\mu X . \lambda b . \lambda u$. case $u$ of & $\nu X . \lambda b . \lambda u$. case $u$ of \\
\hline\langle\rangle$\quad \mapsto \top$ & {$[\langle\rangle \quad \mapsto \perp$} \\
\hline$b^{\prime} \star u \mapsto R\left(b, b^{\prime}\right) \wedge X\left(b^{\prime}, u\right)$ & {$\left[b^{\prime} \star u \mapsto R\left(b, b^{\prime}\right) \vee X\left(b^{\prime}, u\right)\right.$} \\
\hline alignment of $R$ from $b_{0}\left(R_{\top}^{\triangleright}\left(b_{0}\right)\right)$ & blockings of $R$ from $b_{0}\left(R_{\perp}^{\triangleright}\left(b_{0}\right)\right)$ \\
\hline$\lambda u$. case $u$ of & $\lambda u$. case $u$ of \\
\hline$\mapsto \top$ & $\mapsto \perp$ \\
\hline$\mapsto R\left(b_{0}, b\right)$ & $b \quad \mapsto R\left(b_{0}, b\right)$ \\
\hline$u^{\prime} \star b \star b^{\prime} \mapsto R\left(b, b^{\prime}\right)$ & $u^{\prime} \star b \star b^{\prime} \mapsto R\left(b, b^{\prime}\right)$ \\
\hline
\end{tabular}

Table 9: Dependent choice and bar induction principles

\begin{tabular}{|l|l|}
\hline \multicolumn{1}{|c|}{ ill-foundedness-style } & \multicolumn{1}{c|}{ well-foundedness-style } \\
\hline Dependent Choice $\left(\mathrm{DC}_{B R b_{0}}^{\text {serial }}\right)$ & Dual to Dependent Choice $\left(\mathrm{Bl}_{B R b_{0}}^{\text {least }}\right)$ \\
$R$ serial $\Rightarrow R_{\uparrow}^{\triangleright}\left(b_{0}\right)$ has an infinite branch & $R_{\perp}^{\triangleright}\left(b_{0}\right)$ barred $\Rightarrow R$ has a least element \\
\hline
\end{tabular}


Table 10: Logically opposite dual concepts on dual relations

\begin{tabular}{|c|c|}
\hline ill-foundedness-style & well-foundedness-style \\
\hline$R$ left-total & $R$ grounded (*) \\
$\forall a \exists b R(a, b)$ & $\exists a \forall b R(a, b)$ \\
\hline$R$ choosable & $R$ barred \\
$\exists \alpha \forall a \forall b(\alpha(a)=b \Rightarrow R(a, b))$ & $\forall \alpha \exists a \exists b(\alpha(a)=b \wedge R(a, b))$ \\
\hline
\end{tabular}

Table 11: Countable choice and weak bar induction principles

\begin{tabular}{|c|c|}
\hline ill-foundedness-style & well-foundedness-style \\
\hline Countable Choice $\left(\mathrm{CC}_{B R}\right)$ & Dual to Countable Choice $\left(\mathrm{WBI}_{B R}\right)$ \\
$R$ left-total $\Rightarrow R$ choosable & $R$ barred $\Rightarrow R$ grounded \\
\hline
\end{tabular}

Proof: From left to right, we take $R_{T}^{\triangleright}\left(b_{0}\right)$ and use Proposition 11. From right to left, we take $B_{T}$ and $R_{T}$, obtaining \langle\rangle$\in B_{T}$ from $T$ productive. We get an infinite branch $\beta$ of elements of $B_{T}$ such that $u \prec_{s} \beta$ implies $\left(R_{T}\right)_{\top}^{D^{\triangleright}}(\langle\rangle)(u)$, which means first that $R_{T}(\langle\rangle, \beta(0))$, thus $\beta(0)=b$ for some $b$, then, secondly, that for all $n, R_{T}(\beta(n), \beta(n+1))$, i.e. $\beta(n+1)=\beta(n) \star b$ for some $b$. It is then enough to define $\alpha(n)$ to be the corresponding $b$ to get an infinite branch of elements of $B$. Let us now consider $u \prec_{s} \alpha$. We already know \langle\rangle$\in T$ from $T$ productive. Otherwise, for $u$ non empty, we get by induction that $u$ coincides with $\beta(|u|-1)$ which is in $T$ because $u \in B_{T}$ implies $T$ being productive from $u$.

As a final remark, let us mention countable Zorn's lemma [27]: If a partial order $S$ on a countable set has no countable chain, it has a maximal element. It corresponds to the instantiation on $\neg S$ of the generalisation of the scheme $R_{\perp}^{*}\left(b_{0}\right)$ barred implies $R$ has a least element over all $b_{0}$, using our definitions up to classical reasoning, and dropping the partial order requirement. This is the case because a least element is a maximal one in the complement of a relation and because, classically, the barring of all antichainings of $\neg S$ is the same as the absence of countable chains in a partial order $S$.

\subsection{Relation to countable choice}

In Table 10, we introduce definitions allowing to state in Table 11 the axiom of countable choice, CC, and its dual, which we call weak bar induction. Note that left-total and grounded are respective generalisations of serial and having a least element to non-necessarily homogeneous relations.

We shall prove that CC is derivable from DCproductive and introduce for that the alignment of a sequential relation over $\mathbb{N} \times A$ as a predicate over $A^{*}$ (see Table 12). We have:

Theorem 3 For $B$ and $R$ given ( $R$ relation over $\mathbb{N}$ and $B$ ), $C C_{B R}$ is equivalent to $D C_{B R_{\top}^{\mathrm{N}}}^{\text {prodive }}$. Dually, $W B I_{B R}$ is

Table 12: Logically opposite dual concepts on dual relations

\begin{tabular}{|c|c|}
\hline ill-foundedness-style & well-foundedness-style \\
\hline \multicolumn{2}{|c|}{ Intensional concepts } \\
\hline sequential positive alignment of $R\left(R_{\top}^{\mathbb{N}}\right)$ & sequential negative alignment of $R\left(R_{\perp}^{\mathbb{N}}\right)$ \\
\hline$\lambda u$. case $u$ of & $\lambda u$. case $u$ of \\
\hline $\left.\begin{array}{l}\langle\rangle \quad \mapsto \top \\
u \star b \mapsto R(|u|, b)\end{array}\right]$ & {$\left[\begin{array}{l}\langle\rangle \quad \mapsto \perp \\
u \star b \mapsto R(|u|, b)\end{array}\right]$} \\
\hline
\end{tabular}


equivalent to $B P_{B R_{\perp}^{\mathbb{N}}}^{\mathbb{N}}$.

PRoOF: The correspondence between $R$ left-total and $R_{\top}^{\mathbb{N}}$ productive is obtained by coinduction from left to right and, from right to left, by extracting the $n^{\text {th }}$ element of the proof of $R_{\top}^{\mathbb{N}}$ to get the image of $n$ by $R$. The correspondence between $R$ choosable (as a relation) and $R_{\top}^{\mathbb{N}}$ choosable (as a predicate on $B^{*}$ ) is obtained as follows. If $\alpha$ is the witness of $R$ choosable, we define an infinite branch by $\alpha^{\prime}(n) \triangleq(n, \alpha(n))$. Conversely, if $\alpha^{\prime}$ is an infinite branch, we define $\alpha(n) \triangleq b$ whenever $\alpha^{\prime}(n)$ is necessarily of the form $(n, b)$. Similarly for the dual case.

We do not conversely expect to be able in general to express DCproductive in term of CC since countable choice is strictly weaker than dependent choice, and similarly for $\mathrm{BI}^{\text {ind }}$ in terms of WBI. However, if $B$ is countable, the statements of DC and CC become mutually expressible using classical-reasoning-based minimisation: their common strength as choice principle then is not greater than the axiom of unique choice. The latter itself is a tautology if functions are represented as functional relations. It has however the logical effect of reifying functional relations as proper functions if functions are represented as proper objects in a functional type. We conjecture that the equivalence of $\mathrm{BI}^{\text {ind }}$ and WBI with countable codomain is provable intuitionistically.

\section{Non sequential generalisation of dependent choice and bar induction}

In the previous section, we considered predicates branching countably many times over a domain $B$. In this section, we investigate how to generalise countable sequences of branchings to branching in an arbitrary order over a non-necessarily countable domain $A$.

When $B$ is $\mathbb{B}$, we shall obtain principles equivalent to the Boolean Prime Ideal/Filter Theorem (ill-founded case), or to the Completeness Theorem but we shall recover the strength of dependent choice (ill-founded case) and bar induction (well-founded case) when $A$ is countable. In particular we will obtain the strength of the Weak Fan Theorem (well-founded case) and Weak König's Lemma (ill-founded case), up to classical reasoning, when $A$ is countable and $B$ is $\mathbb{B}$.

For a certain instance, we will get the strength of the full axiom of choice. However, the new principle is limited. For instance, for $A \triangleq \mathbb{B}^{\mathbb{N}}$ and $B \triangleq \mathbb{N}$, we end up with an inconsistent axiom.

\subsection{Finite approximations of functions}

Let $A$ be a domain whose elements are ranged over by the letters $a, a^{\prime}, \ldots$ and $B$ a codomain whose elements are ranged over by the letters $b, b^{\prime}, \ldots$ Let $T$ be a predicate over $(A \times B)^{*}$ i.e. over sequences of pairs in $A$ and $B$, thought as a set of possible finite approximations of a function from $A$ to $B$. We use $v$ to range over approximations.

We order $(A \times B)^{*}$ by set inclusion, which we write $\subseteq$. We overload the notations $\downarrow^{-} T$ and $\uparrow^{+} T$ to now respectively mean the downwards closure under restriction and upwards closure under extension with respect to $\subseteq$. In particular, since $v \subseteq v^{\prime}$ for any $v^{\prime}$ obtained from $v$ by permutation or duplication, $\downarrow^{-} T$ and $\uparrow+T$ are stable by permutation. We write $v \sim v^{\prime}$ for $v \subseteq v^{\prime}$ and $v^{\prime} \subseteq v$, i.e. for the equivalence of $v$ and $v^{\prime}$ as finite sets.

Note that we do not prevent that a sequence may contain several occurrences of the same pair $(a, b)$. However, such a sequence shall be equivalent to a sequence without redundancies (this design choice is somewhat arbitrary, we just found it more convenient not to enforce the absence of redundancies).

We write $(a, b) \in v$ to mean that $(a, b)$ is one of the elements of the sequence. For $v \in(A \times B)^{*}$, we write $d o m(v)$ for the set of $a$ such that there is some $b$ such that $(a, b) \in v$. For $\alpha \in A \rightarrow B$ and $v \in(A \times B)^{*}$, we define $v \prec \alpha$ to mean $\alpha(a)=b$ for all $(a, b) \in v$, or more formally for the predicate defined by the following clauses:

$$
\overline{\langle\rangle \prec \alpha} \quad \frac{v \prec \alpha \quad \alpha(a)=b}{v \star(a, b) \prec \alpha}
$$

We think of $(A \times B)^{*}$ as finite approximations of functions from $A$ to $B$ and of predicates over finite approximations as constraints generating an ideal or a filter.

In Table 13, we generalise the notion of productive over (morally) trees into a coinductive notion of $A$ - $B$-approximable relative to a valid finite set of approximations, and dually, we generalise the notion of inductively barred from holding on a sequence to holding relative to a finite set of approximations.

\subsection{Generalised Dependent Choice and Generalised Bar Induction}

We state the generalisation of dependent choice and bar induction to non-sequential choices over a non-necessarily countable domain in Table 14. Called GDC $A B T$ (shortly $\mathrm{GDC}_{A B}$ or GDC as schemes) and $\mathrm{GBI}_{A B T}$ (shortly GBI$A B$ or GBI as schemes), 
Table 13: Logically opposite dual concepts on dual predicates

\begin{tabular}{|c|c|}
\hline ill-foundedness-style & well-foundedness-style \\
\hline \multicolumn{2}{|c|}{ Intensional concepts } \\
\hline $\begin{array}{l}T A \text {-B-approximable from } v \\
\nu X . \lambda v .\left(\begin{array}{l}v \in \downarrow^{-} T \wedge \\
\forall a \notin \operatorname{dom}(v) \\
\exists b(v \star(a, b) \in X)\end{array}\right)\end{array}$ & $\begin{array}{r}T \text { inductively } A \text { - } B \text {-barred from } v \\
\mu X . \lambda v \cdot\left(\begin{array}{l}v \in \uparrow+T \vee \\
\exists a \notin \operatorname{dom}(v) \\
\forall b(v \star(a, b) \in X)\end{array}\right)\end{array}$ \\
\hline $\begin{array}{c}T A \text { - } B \text {-approximable } \\
T A \text { - } B \text {-approximable from }\langle\rangle\end{array}$ & $\begin{array}{c}T \text { inductively } A \text { - } B \text {-barred } \\
T \text { inductively } A \text { - } B \text {-barred from }\langle\rangle\end{array}$ \\
\hline \multicolumn{2}{|c|}{ Extensional concepts } \\
\hline $\begin{array}{c}T \text { has an } A \text { - } B \text {-choice function } \\
\quad \exists \alpha \forall u(u \prec \alpha \Rightarrow u \in T)\end{array}$ & $\begin{array}{c}T \text { is } A \text { - } B \text {-barred } \\
\forall \alpha \exists u(u \prec \alpha \wedge u \in T)\end{array}$ \\
\hline
\end{tabular}

Table 14: Dual axioms on dual predicates

\begin{tabular}{|l|l|}
\hline \multicolumn{1}{|c|}{ ill-foundedness-style } & \multicolumn{1}{c|}{ well-foundedness-style } \\
\hline Generalised Dependent Choice $\left(\mathrm{GDC}_{A B T}\right)$ & Generalised Bar Induction $\left(\mathrm{GBI}_{A B T}\right)$ \\
$T A$ - $B$-approximable $\Rightarrow T$ has an $A$ - $B$-choice function & $T A$-B-barred $\Rightarrow T$ inductively $A$ - $B$-barred \\
\hline
\end{tabular}

they are generalisations in the sense that they respectively capture DCproductive and $\mathrm{Bl}^{\text {ind }}$ for countable $A$, where by countable is meant the existence of a bijection between $A$ and $\mathbb{N}$.

To prove it, let us assume without loss of generality that $A$ is $\mathbb{N}$ itself. We say that $v \in(\mathbb{N} \times B)^{*}$ is sequential whenever either $v$ is empty or $v$ has the form $v^{\prime} \star\left(\left|v^{\prime}\right|, b\right)$ with $v^{\prime}$ itself sequential. To each $u \in B^{*}$ we can associate a sequential element $\operatorname{ord}(u)$ by $\operatorname{ord}(\emptyset) \triangleq \emptyset$ and $\operatorname{ord}(u \star b) \triangleq \operatorname{ord}(u) \star(|u|, b)$. We then extend $\subseteq$ from a relation on $(\mathbb{N} \times B)^{*}$ to an heterogeneous relation on $(\mathbb{N} \times B)^{*}$ and $B^{*}$ by writing $v \subseteq u$ to mean $v \subseteq$ ord $(u)$.

To each $T$ over $(\mathbb{N} \times B)^{*}$, we can associate $\|T\|$ on $B^{*}$ by $u \in\|T\| \triangleq \operatorname{ord}(u) \in T$. Conversely, to each $T$ over $B^{*}$, we can associate $\widehat{T}$ on $(\mathbb{N} \times B)^{*}$ defined by $v \in \widehat{T} \triangleq \exists u \in T(v=\operatorname{ord}(u))$. We have:

Proposition 12 For $T$ over $B^{*}, u \in T$ iff $u \in\|\widehat{T}\|$

ProOF: Direct by definition.

Proposition 13 For $T$ over $(\mathbb{N} \times B)^{*}, T$ is $\mathbb{N}$-B-approximable iff $\|T\|$ is productive, and, for $T$ over $B^{*}, \widehat{T}$ is $\mathbb{N}$ - $B$ approximable iff $T$ is productive. Dually, $T$ is inductively $\mathbb{N}$-B-barred iff $\|T\|$ is inductively barred, and, $\widehat{T}$ is inductively $\mathbb{N}$-B-barred iff $T$ is inductively barred.

Proof: By duality and Proposition 12, it is enough to prove the first item. The proof is by coinduction in both directions.

From left to right, we prove $T \mathbb{N}$ - $B$-approximable from $\operatorname{ord}(u)$ implies $\|T\|$ productive from $u$. We take $|u|$ for $a$ in the definition of $\mathbb{N}$ - $B$-approximable from $\operatorname{ord}(u)$, get some $b$ and pass it to the definition of $\|T\|$ productive from $u$.

From right to left, we prove more generally that if $\|T\|$ is productive from $u$ then $T$ is $\mathbb{N}$ - $B$-approximable from $v$ for all $v \subseteq u$. By definition of $u \in\|T\|$, we have $\operatorname{ord}(u) \in T$ and thus $v \in \downarrow^{-} T$ by closure of $T$. Now, take $n \notin d o m(v)$. If $n<|u|$, we set $b$ to be $u(n)$ and apply the coinduction hypothesis with $v$ extended with $b$, which still satisfies $v \star b \subseteq u$ by a combinatorial argument. If $n \geq|u|$, we explore the proof of productivity of $\|T\|$ one step further, getting some $\bar{b}$ such that $u \star b \in\|T\|$ and $\|T\|$ is productive from $u \star b$. The property $v \subseteq(u \star b)$ continues to hold and we reason by induction on $|u|-n$ until falling into the first case.

Similarly, we have: 
Table 15: Logically opposite dual concepts on dual homogeneous relations

\begin{tabular}{|c|c|}
\hline ill-foundedness style & well-foundedness-style \\
\hline \multicolumn{2}{|c|}{ Intensional concepts } \\
\hline positive alignment of $R\left(R_{\top}\right)$ & negative alignment of $R\left(R_{\perp}\right)$ \\
\hline$\lambda u$. case $u$ of & $\lambda u$. case $u$ of \\
\hline\langle\rangle$\quad \mapsto \top$ & \langle\rangle$\quad \mapsto \perp$ \\
\hline$u \star(a, b) \mapsto R(a, b)$ & $u \star(a, b) \mapsto R(a, b)$ \\
\hline
\end{tabular}

Table 16: The axiom of the Choice and its dual

\begin{tabular}{|l|l|}
\hline \multicolumn{1}{|c|}{ ill-foundedness-style } & \multicolumn{1}{c|}{ well-foundedness-style } \\
\hline Standard Axiom of Choice $\left(\mathrm{AC}_{A B R}\right)$ & Dual to Standard Axiom of Choice $\left(\right.$ co- $\left.\mathrm{AC}_{A B R}\right)$ \\
$R$ left-total $\Rightarrow R$ has an $A$ - $B$-choice function & $R A$ - $B$-barred $\Rightarrow R$ has a least element \\
\hline
\end{tabular}

Proposition $14 \downarrow^{-} T$ has an $\mathbb{N}$-B-choice function iff $\|T\|$ has an infinite branch, and, $\downarrow^{-} \widehat{T}$ has a $\mathbb{N}$-B-choice function iff $T$ has an infinite branch. Dually, $\uparrow^{+} T$ is barred iff $\|T\|$ is $B$-barred, and, $\uparrow^{+} \widehat{T}$ is $\mathbb{N}$-B-barred iff $T$ is barred.

Proof: By duality and Proposition 12, it is enough to prove the first item. From left to right, if $u \prec_{s} \alpha$, it is enough to consider $\operatorname{ord}(u) \prec \alpha$. From right to left, if $v \prec \alpha$, we consider $u \triangleq \alpha_{n}$ where $n$ is $|v|$. We have $u \prec_{s} \alpha$ thus $u \in T$ and $\operatorname{ord}(u) \in T$. Since $v \subseteq \operatorname{ord}(u)$, we get $v \in \downarrow^{-} T$.

Consequently, we have:

Proposition $15 D C_{B}^{\text {productive }}$ iff $G D C_{\mathbb{N} B}$ and $B i_{B}^{\text {ind }}$ iff $G B /_{\mathbb{N} B}$.

Proof: We mediate by the property that $\mathrm{GDC}_{\mathbb{N} B T}$ is equivalent as a scheme to " $T A$ - $B$-approximable implies $\downarrow^{-} T$ has an $A$ - $B$-choice function". In one direction this is the case because $\downarrow^{-} T \subseteq T$, in the other direction, because $\downarrow^{-}$is idempotent. The other equivalence holds by duality.

\subsection{Inconsistency of the unconstrained form of Generalised Dependent Choice and Gen- eralised Bar Induction}

In its full generality, the generalisation of GDC and GBI obtained by allowing non-countable branchings over an arbitrary codomain $B$ is inconsistent: for large enough $A$ and $B$, it may happen that some $T$ is coinductively $A$ - $B$-approximable without $T$ having a (full) $A$-B-choice function. Indeed, take $A \triangleq \mathbb{B}^{\mathbb{N}}$ and $B \triangleq \mathbb{N}$ and filter the choice function so that it is injective. That is, we define $u \in T$ as follows: if $u$ is \langle\rangle or $u$ is a singleton then $u \in T$ holds; if $u$ is $u^{\prime} \star(f, n) \star\left(f^{\prime}, n^{\prime}\right)$ with $f$ extensionally different from $f^{\prime}$ then $n \neq n^{\prime}$.

Then, $T$ is coinductively $\mathbb{B}^{\mathbb{N}}-\mathbb{N}$-approximable by successively extending $u$ with $(f,|u|)$ for any $f$ not already in $\operatorname{dom}(u)$. But there is no total choice function $\alpha$ from $\mathbb{B}^{\mathbb{N}}$ to $\mathbb{N}$, since, by Cantor's theorem, such a function is necessarily noninjective. Thus, taking $f$ and $f^{\prime}$ distinct such that $n \triangleq \alpha(f)=\alpha\left(f^{\prime}\right)$, we get that the sequence $(f, n),\left(f^{\prime}, n\right) \prec \alpha$ is not in $T$.

Therefore, we have:

Proposition $16 G D C_{\mathbb{B}^{\mathbb{N}}}$ and $\left.G B\right|_{\mathbb{B}^{N} \mathbb{N}}$ are inconsistent.

\subsection{Relation to the general axiom of choice}

We state the standard axiom of choice in Table 16 and prove that it is equivalent to an instance of the generalised dependent choice GDC. To do so, we generalise in Table 15 the notion of sequential alignment introduced in Section 2.8 to the notion of alignment of a relation on $A \times B$ as a predicate over $(A \times B)^{*}$. 
Table 17: Dual axioms on dual predicates

\begin{tabular}{|l|l|}
\hline \multicolumn{1}{|c|}{ ill-foundedness-style } & \multicolumn{1}{|c|}{ well-foundedness-style } \\
\hline Generalised Weak Kônig's Lemma $\left(\mathrm{GDC}_{A \mathbb{B} T}\right)$ & Generalised Weak Fan Theorem $\left(\mathrm{GBI}{ }_{A \mathbb{B} T}\right)$ \\
$T A$ - $\mathbb{B}$-approximable $\Rightarrow T$ has an $A$ - $\mathbb{B}$-choice function & $T A$ - $\mathbb{B}$-barred $\Rightarrow T$ inductively $A$ - $\mathbb{B}$-barred \\
\hline
\end{tabular}

Theorem $4 A C_{A B R}$ is logically equivalent to $G D C_{A B R_{\top}}$

Proof: The proof is similar to the one of Theorem 3. It consists in first showing that any partial choice function $\alpha$ is actually total for a left-total $R$. Indeed, for $a \in A$, there is a $b$ such that $R(a, b)$ by left-totality. Thus, from any $u$ such that $u \in R_{\top}$ and $u \star(a, b) \notin R_{\top}$, we get a contradiction by definition of $R_{\top}$.

\section{The Boolean instances of generalised dependent choice and bar induction: relation to the Boolean Prime Ideal/Filter Theorem and completeness theorems}

\subsection{Generalised Weak König Lemma and Generalised Weak Fan Theorem}

By instantiating the codomain $B$ to $\mathbb{B}$ in $\mathrm{GDC}$ and $\mathrm{GBI}$, we obtain extensions $\mathrm{GBI}_{A \mathbb{B}}$ of the Weak Fan Theorem (more precisely of $B I_{\mathbb{B}}^{\text {ind }}$ ) and $G_{D C}$ of the Weak Kónig Lemma (more precisely of $D C_{\mathbb{B}}^{\text {productive }}$ ) which replace the countable sequence of branching made on a "tree" (in practise predicates) by a countable sequence of choices in arbitrary order over a non-necessarily countable domain. This shall be proved classically equivalent to the Boolean Prime Ideal/Filter Theorem. This is consistent with the standard reverse mathematics results which show that the completeness theorem is equivalent to the Weak Kônig's Lemma on countable theories [24] but equivalent to the Boolean Prime Filter Theorem on theories of arbitrary cardinality $[13,14,18,11]$.

\subsection{Logical reading: relation to completeness theorem}

We can give a logical reading to $(A \times \mathbb{B})^{*}$ as follows. We call atom any element of $A$. We interpret pairs in $A \times \mathbb{B}$ as literals, i.e. as atoms together with a polarity indicating whether the atom is negated or not (we adopt the convention that 1 stands for non-negated and 0 for negated). We call clause any unordered sequence of elements in $A \times \mathbb{B}$. We call context any unordered sequence of elements of $A$. We range over clauses by the letters $C, D$ and over contexts by the letters $\Gamma, \Delta, \ldots$

Any clause $C$ can canonically be represented as a pair of two contexts $\Gamma$ and $\Delta$ with $\Gamma$ the subset of non-negated elements of $A$ in $C$ and $\Delta$ the subset of negated elements. We write $\Gamma \triangleright \Delta$ for such a pair. We call a set of clauses a theory and use the letter $\mathcal{T}$ to range over theories. We write $(\Gamma \triangleright \Delta) \in \mathcal{T}$ to mean that there is a clause of $\mathcal{T}$ associated to the pair $\Gamma \triangleright \Delta$. We write $\Gamma \nmid \Delta$ to mean that $\Gamma$ and $\Delta$ have an atom in common.

We consider (a variant of) Scott's notion of entailment relation [23], i.e. of a preorder relation up to "side contexts". Let $\mathcal{T}$ be a theory on $A$. We define the entailment relation generated by $\mathcal{T}$ to be the smallest relation on sequents, written $\Gamma \vdash_{\mathcal{T}} \Delta$, such that the following holds:

$$
\frac{\Gamma \ell \Delta}{\Gamma \vdash_{\mathcal{T}} \Delta} \mathrm{Ax} \frac{(\Gamma \triangleright \Delta) \in \mathcal{T}^{+} \mathcal{T}}{\Gamma \vdash_{\mathcal{T}} \Delta} \mathrm{Ax}_{T} \frac{\Gamma \vdash_{\mathcal{T}} \Delta, F \quad \Gamma, F \vdash_{\mathcal{T}} \Delta}{\Gamma \vdash_{\mathcal{T}} \Delta} \mathrm{Cut}
$$

It is usual to add an explicit weakening rule to the definition of entailment relation but here we shall consider it as an admissible rule. Formally, the existence of a derivation of $\Gamma \vdash_{\mathcal{T}} \Delta$ using the inferences rules above is the same as

$$
\vdash_{\mathcal{T}} \triangleq \mu X . \lambda(\Gamma \triangleright \Delta) .\left(\begin{array}{l}
(\Gamma \triangleright \Delta) \in \mathcal{T}^{+} \mathcal{T} \\
\left.\vee \exists F \notin(\Gamma \cup \Delta)\left(\begin{array}{l}
(\Gamma, F \triangleright \Delta) \in X \\
\wedge(\Gamma \triangleright \Delta, F) \in X
\end{array}\right)\right)
\end{array}\right)
$$

Thus, $\Gamma \vdash_{\mathcal{T}} \Delta$ exactly says that $\mathcal{T}$ is inductively $A$-BB-barred from $\Gamma \triangleright \Delta$. 
Table 18: Logically opposite dual concepts of logic on the same predicate

\begin{tabular}{|c|c|}
\hline ill-foundedness-style & well-foundedness-style \\
\hline \multicolumn{2}{|c|}{ Intensional concepts } \\
\hline $\mathcal{T}$ is (positively) consistent & $\mathcal{T}$ is inconsistent \\
$\forall_{\mathcal{T}}$ & $\vdash_{\mathcal{T}}$ \\
\hline \multicolumn{2}{|c|}{ Extensional concepts } \\
\hline $\mathcal{T}$ has a model & $\mathcal{T}$ is unsatisfiable \\
$\exists \alpha \alpha \vDash \mathcal{T}$ & $\forall \alpha \alpha \not \mathcal{T}$ \\
\hline
\end{tabular}

Table 19: Reformulation of Table 17 as statements about a given logical theory

\begin{tabular}{|l|l|}
\hline \multicolumn{1}{|c|}{ ill-foundedness-style } & \multicolumn{1}{|c|}{ well-foundedness-style } \\
\hline Existence-style Completeness Theorem $\left(\mathrm{Compl}^{-}(A \mathcal{T})\right)$ & Provability-style Completeness Theorem $\left(\mathrm{Compl}^{+}(A \mathcal{T})\right)$ \\
$\mathcal{T}$ consistent $\Rightarrow \mathcal{T}$ has a model & $\mathcal{T}$ unsatisfiable $\Rightarrow \mathcal{T}$ inconsistent \\
\hline
\end{tabular}

Conversely, let us consider $\Gamma \nvdash_{\mathcal{T}} \Delta$. We could define it by negation of $\Gamma \vdash_{\mathcal{T}} \Delta$ but we instead give a direct explicit definition which we call positive disprovability. Let $\mathcal{T}^{C}$ denote the complement of $\mathcal{T}$. The positive disprovability $\Gamma \forall \mathcal{C} \Delta$ can be characterised as the $\mathcal{T}^{C} A$ - $\mathbb{B}$-approximability from $\Gamma \triangleright \Delta$, that is, formally:

$$
(\Gamma \triangleright \Delta) \in \nu X . \lambda(\Gamma \triangleright \Delta) .\left(\begin{array}{l}
(\Gamma \triangleright \Delta) \in \downarrow^{-} \mathcal{T}^{C} \\
\wedge \forall F \notin(\Gamma \cup \Delta)\left(\begin{array}{l}
(\Gamma, F \triangleright \Delta) \in X \\
\vee(\Gamma \triangleright \Delta, F) \in X
\end{array}\right)
\end{array}\right)
$$

Let $\alpha$ be a function from $A$ to $\mathbb{B}$. It can be interpreted as a model over $A$ with 1 to indicate that the atom is true in the model and 0 to indicate that the atom is false in the model.

Truth $\alpha \vDash \mathcal{T}$ of a theory $\mathcal{T}$ in a model $\alpha$ can be defined by

$$
\alpha \vDash \mathcal{T} \triangleq \forall(\Gamma \triangleright \Delta) \in \mathcal{T}(\Gamma \subset \alpha \Rightarrow \Delta \gamma \alpha)
$$

where we use the notation $\Gamma \subset \alpha$ to mean that $\forall a \in \Gamma \alpha(a)=1$ and the notation $\Delta \nmid \alpha$ to mean $\neg \forall a \in \Delta \alpha(a)=0$. Then, $\mathcal{T}$ has a model if there exists $\alpha$ such that $\alpha \vDash \mathcal{T}$.

Falsity $\alpha \not \models \mathcal{T}$ of a theory $\mathcal{T}$ in a model $\alpha$ can be defined explicitly by

$$
\alpha \not \forall \mathcal{T} \triangleq \exists(\Gamma \triangleright \Delta) \in \mathcal{T} \Gamma \subset \alpha \wedge \Delta \subset \bar{\alpha}
$$

where $\Delta \subset \bar{\alpha}$ stands for $\forall a \in \Delta \alpha(a)=0$. The theory $\mathcal{T}$ is unsatisfiable if for all $\alpha, \alpha \not \forall \mathcal{T}$.

Then, we get that $\mathcal{T} A$-BB-barred corresponds exactly to the unsatisfiability of $\mathcal{T}$. Also, noticing that, $\exists \alpha \forall u(u \prec$ $\left.\alpha \Rightarrow u \in \mathcal{T}^{C}\right)$ is linearly equivalent to $\exists \alpha \forall u(u \in \mathcal{T} \Rightarrow \neg u \prec \alpha)$ and that $\neg u \prec \alpha$ is linearly equivalent to $\left.\Gamma \subset \alpha \Rightarrow \Delta\right\rangle \alpha$, we get that $\mathcal{T}^{C}$ has an $A$-BB-choice function if and only if there exists a model for $\mathcal{T}$ (see Table 18 where $\vdash_{\mathcal{T}}$ and $\forall_{\mathcal{T}}$ refer to the provability and positive disprovability of the empty clause).

The completeness theorem of logic is conventionally expressed either as the existence of a model for any consistent theory, or contrapositively, that if a theory is unsatisfied in all theories, then it is inconsistent, as shown on Table 19.

For instance, see Rinaldi, Schuster and Wessel [22] for the statement of a completeness theorem such as $\operatorname{Compl}^{+}(\mathcal{T})$, up to the identification of some $\exists$ with $\neg \neg \exists$. See also e.g. [21] for an algebraic reading.

Summing up, we have:

Generalised Weak König's Lemma over $\mathcal{T}^{C}$, i.e. $\mathrm{GDC}_{A \mathbb{B} \mathcal{T}^{C}}$, logically reads as $\mathrm{Compl}^{-}(A \mathcal{T})$

Generalised Weak Fan Theorem over $\mathcal{T}$, i.e. $\mathrm{GBI}_{A \mathbb{B} \mathcal{T}}$, logically reads as $\mathrm{Compl}^{+}(A \mathcal{T})$

where it should be reminded that, to preserve the duality, we used in $\operatorname{Compl}^{-}(\mathcal{T})$ an explicit definition of $\Gamma \nvdash_{\mathcal{T}} \Delta$ which is classically equivalent to but intuitionistically stronger than the negation of $\Gamma \vdash_{\mathcal{T}} \Delta$. 
Table 20: Reformulation of Table 17 as statements about a given Boolean algebra

\begin{tabular}{|l|l|}
\hline \multicolumn{1}{|c|}{ ill-foundedness-style } & \multicolumn{1}{c|}{ well-foundedness-style } \\
\hline Boolean Prime Filter Theorem $\left(\mathrm{BPF}_{\mathcal{B}}(F)\right.$ for $F$ a filter $)$ & "Boolean Full Filter Theorem" (co-BPF $(F)$ for $F$ a filter $)$ \\
$F$ proper $\Rightarrow F$ extensible into a prime filter & $F$ not extensible into a prime filter $\Rightarrow F$ full \\
\hline Boolean Prime Ideal Theorem $\left(\mathrm{BPI}_{\mathcal{B}}(I)\right.$ for $I$ an ideal $)$ & "Boolean Full Ideal Theorem" (co-BPI ${ }_{\mathcal{B}}(I)$ for $I$ an ideal $)$ \\
$I$ proper $\Rightarrow I$ extensible into a prime ideal & $I$ not extensible into a prime ideal $\Rightarrow I$ full \\
\hline
\end{tabular}

\subsection{Algebraic reading: relation to the Boolean Prime Ideal/Filter Theorem}

The previous reasoning based on entailment relations can also be expressed in terms of Boolean algebras, connecting Generalised Weak König's Lemma to the Boolean Prime Ideal/Filter Theorem. There is however a caveat: the standard definition of proper filter and proper ideal relies on an intrinsically classical definition, so that the relation holds only with a classical reading of the connectives involved in the definition of approximable.

Let $(\mathcal{B}, \dot{\vee}, \dot{\wedge}, \dot{\perp}, \dot{\top}, \dot{ᄀ})$ be a Boolean algebra and $\dot{\vdash}$ the canonical order relation associated to it $\left(b \dot{\vdash} b^{\prime} \triangleq b \dot{\wedge} b^{\prime}=b\right)$. We call filter over $\mathcal{B}$ any non-empty subset $F$ of $\mathcal{B}$ which is closed under $\dot{\wedge}$ and closed under $\dot{ } \boldsymbol{~ o n ~ t h e ~ r i g h t . ~ A ~ f i l t e r ~ i s ~ p r o p e r ~}$ if it does not contain $\dot{\perp}$. Otherwise, it coincides with $\mathcal{B}$ and we call it full. We call ultrafilter a maximal proper filter. A maximal filter in a Boolean algebra can be described as a map $U$ from $\mathcal{B}$ to $\mathbb{B}$ such that $b_{1} \wedge b_{2} \in U$ iff $b_{1} \in U \wedge b_{2} \in U$, $b_{1} \dot{\vee} b_{2} \in U$ iff $b_{1} \in U \vee b_{2} \in U, \dot{\neg} b \in U$ iff $\neg(b \in U), \dot{\top} \in U$, and $\dot{\perp} \notin U$. In a Boolean algebra, the notion of maximal filter coincides with the notion of prime filter where a filter $F$ is prime if $\left(b_{1} \dot{\vee} b_{2}\right) \in F$ implies $b_{1} \in F$ or $b_{2} \in F$.

Dually, we call ideal over $\mathcal{B}$ any non-empty subset $I$ of $\mathcal{B}$ which is closed under $\dot{V}$ and closed under $\dot{\vdash}$ on the left. An ideal is proper if it does not contain $\dot{\top}$, and full otherwise. A prime ideal $I$ is such that $\left(b_{1} \dot{\wedge} b_{2}\right) \in I$ implies $b_{1} \in I$ and $b_{2} \in I$ and this coincides with the notion of maximal proper ideal. A prime/maximal proper ideal can be characterised the same way as a prime/maximal proper filter, i.e. as a map $U$ from $\mathcal{B}$ to $\mathbb{B}$ such that $b_{1} \wedge b_{2} \in U$ iff $b_{1} \in U \wedge b_{2} \in U$, $b_{1} \dot{\vee} b_{2} \in U$ iff $b_{1} \in U \vee b_{2} \in U, \dot{\neg} b \in U$ iff $\neg(b \in U)$, except that this time $\dot{\perp} \in U$ and $\dot{\top} \notin U$.

There is a family of provably equivalent theorems about the existence of maximal/prime ideals/filters in Boolean algebras (see e.g. Jech [18, 2.3]) called Boolean Prime Ideal Theorem in arbitrary Boolean algebras, or Ultrafilter Theorem in the Boolean algebra of subsets of a set. We consider in Table 20 the case of a general Boolean algebra and state the Boolean Prime Ideal Theorem in its two "ideal" and "filter" flavours. We also consider their contrapositive.

We now show that the Boolean Prime Ideal and Prime Filter Theorems and Generalised Weak Kônig's Lemma, i.e. $\mathrm{GDC}_{A \mathbb{B}}$, are mutual instances of each other. We first show that the Generalised Weak König's Lemma is an instance of the Boolean Prime Ideal and Boolean Prime Filter Theorems.

To any domain $A$ we can associate a freely generated Boolean algebra $(\operatorname{Free}(A), \dot{\vee}, \dot{\wedge}, \dot{\perp}, \dot{\top}, \dot{ᄀ})$ by considering the set of algebraic expressions built from $\dot{V}, \dot{\wedge}, \dot{\mathcal{L}}, \dot{\boldsymbol{T}}$ and $\dot{\mathcal{h}}$, all quotiented by the axioms of a Boolean algebra, namely by:

$$
\begin{aligned}
& a \dot{\vee}(b \dot{\vee} c)=(a \dot{\vee} b) \dot{\vee} c \quad a \dot{\wedge}(b \dot{\wedge} c)=(a \dot{\wedge} b) \dot{\wedge} c \\
& a \dot{\vee} b \quad=b \dot{\vee} a \quad a \dot{\wedge} b=b \dot{\wedge} a \\
& a \dot{\vee}(a \dot{\wedge} b)=a \quad a \dot{\wedge}(a \dot{\vee} b)=a \\
& a \dot{\vee} \dot{\perp}=a \quad a \dot{\wedge} \dot{\top}=a \\
& a \dot{\vee}(b \dot{\wedge} c)=(a \dot{\vee} b) \dot{\wedge}(a \dot{\vee} c) \quad a \dot{\wedge}(b \dot{\vee} c)=(a \dot{\wedge} b) \dot{\vee}(a \dot{\wedge} c) \\
& a \dot{\vee} \dot{\neg} a=\dot{\top} \quad a \dot{\wedge} \dot{\sim} a=\dot{\perp}
\end{aligned}
$$

As in the previous section, any $v$ in $(A \times \mathbb{B})^{*}$, can be written under the form $\Gamma \triangleright \Delta$ and two interpretations in $\operatorname{Free}(A)$ are now possible. The first one is to interpret a sequent $\Gamma \triangleright \Delta$ as the formula $(\dot{\bigvee} \neg \Gamma) \dot{V}(\dot{\bigvee} \Delta)$ and to interpret any predicate over $(A \times \mathbb{B})^{*}$ seen as a logical theory $\mathcal{T}$ as the corresponding subset $\mathcal{T}^{\vdash}$ of formulas of this form in $\operatorname{Free}(A)$. It is then standard that the set $F_{\mathcal{T}}$ of logical consequences of $\mathcal{T}^{\vdash}$ defined by $b \in F_{\mathcal{T}} \triangleq \mathcal{T}^{\vdash} \vdash b$ is a filter.

There is also a dual interpretation of $\Gamma \triangleright \Delta$ as the formula $(\dot{\Lambda} \Gamma) \dot{\Lambda}(\dot{\Lambda} \Delta)$ leading to interpret $\mathcal{T}$ as a "theory" of consequences, say ${ }^{\vdash} \mathcal{T}$. Then, the set $I_{\mathcal{T}}$ of logically stronger assumptions of ${ }^{\vdash} \mathcal{T}$ defined by $b \in I_{\mathcal{T}} \triangleq b \vdash \vdash \mathcal{T}$ is an ideal.

This can be rephrased in the following statement where $\overline{\mathcal{T}}$ swaps the role of 0 and 1 in $\mathcal{T}$, i.e. $v \in \overline{\mathcal{T}} \triangleq \bar{v} \in \mathcal{T}$, where $\bar{v}$ itself turns each $(a, 0)$ in $v$ into $(a, 1)$ and vice-versa.

For $\mathcal{T}$ a theory and $\vdash_{\mathcal{T}}$ the entailment relation canonically built from it, it is standard that the set of logical consequences of $\mathcal{T}^{\vdash}$ in $\operatorname{Free}(A)$ forms a filter and the set of logically stronger assumptions of ${ }^{\vdash} \mathcal{T}$ in $\operatorname{Free}(A)$ forms an ideal. In particular, 
the filter is proper if $\mathcal{T}^{\vdash} \forall \perp$, that is if $\mathcal{T}$ is consistent, that is, by Section 4.2, if $\mathcal{T}^{C}$ is $A$-BB-approximable where the connectives involved in the definition of $A$ - $\mathbb{B}$-approximable are interpreted classically. Similarly, the ideal is proper if $\top \forall^{\vdash} \mathcal{T}$, that is if $\overline{\mathcal{T}}$ is consistent, that is if $\overline{\mathcal{T}}^{C}$ is $A$ - $\mathbb{B}$-approximable where, again, the connectives are interpreted classically.

Similarly, we have the general result that prime filters and prime ideals on a free Boolean algebra, here Free $(A)$, are characterised by their intersection with generators, here $A$. Whether other elements of Free $(A)$ belong or not to a prime filter or prime ideal is canonically determined ${ }^{8}$ by:

$$
\begin{array}{llr}
\alpha\left(a \dot{\vee} a^{\prime}\right)=b^{\prime \prime} & \triangleq & \alpha(a)=b \wedge \alpha\left(a^{\prime}\right)=b^{\prime} \wedge b^{\prime \prime}=b+b^{\prime} \\
\alpha\left(a \dot{\wedge} a^{\prime}\right)=b^{\prime \prime} & \triangleq & \alpha(a)=b \wedge \alpha\left(a^{\prime}\right)=b^{\prime} \wedge b^{\prime \prime}=b \cdot b^{\prime} \\
\alpha(\dot{\perp})=b & \triangleq & \text { for ideals } \\
\alpha(\dot{T})=b & \triangleq b=0 & \text { for ideals } \\
\alpha(\dot{\perp})=b & \triangleq b=1 & \text { for filters } \\
\alpha(\dot{T})=b & \triangleq b=0 & \text { for filters } \\
\alpha(\dot{\neg} a)=b^{\prime} & \triangleq &
\end{array}
$$

where $+, \cdot,-$ are the corresponding operations on $\mathbb{B}$.

In particular, the existence of a function from $A$ to $\mathbb{B}$ characterising a prime filter that extends the filter $F_{\mathcal{T}}$ on $\operatorname{Free}(A)$ is the same, by Section 4.2, as a model of $\mathcal{T}$ and as an $A$ - $\mathbb{B}$-choice function for $\mathcal{T}$. Similarly, the existence of a function from $A$ to $\mathbb{B}$ characterising a prime ideal that extends the ideal $I_{\mathcal{T}}$ on $\operatorname{Free}(A)$ is the same as a model of $\overline{\mathcal{T}}$ and as an $A$ - $\mathbb{B}$ choice function for $\overline{\mathcal{T}}$.

Consequently, we get the following:

Theorem $5 G D C_{A \mathbb{B} \mathcal{T}^{C}}$, where the connectives are interpreted classically, is equivalent to $B P F_{\mathrm{Free}(A)}\left(F_{\mathcal{T}}\right)$ and $B P I_{\mathrm{Free}(A)}\left(I_{\overline{\mathcal{T}}}\right)$. On its side, $G B I_{A \mathbb{B} \mathcal{T}}$ is equivalent to $\operatorname{co}_{-B P F_{\mathrm{Free}}(A)}\left(F_{\mathcal{T}}\right)$ and $c \mathrm{co}-\left.B P\right|_{\text {Free }(A)}\left(I_{\overline{\mathcal{T}}}\right)$, even with a linear or intuitionistic interpretation of the connectives.

\section{$5 \quad$ Further questions}

While we did not explicitly state and check it, we suspect that most of our proofs are essentially expressible in a contractionfree logic such as relevant logic or a linear logic without contraction [12], to the notable exception of Proposition 6. Might such a careful check be the purpose of future work.

There is a rich literature on choice axioms and on principles equivalent to choice axioms. Not all of them can be classifyed as either ill- or barred/well-foundedness-style, though. For instance, open induction and update induction [20, $8,6]$, are classically equivalent to bar induction and dependent choice but are formulated as well-foundedness of some order on functions.

The study could also be extended to choice principles such as Zorn's lemma, the ordinal variants of the axiom of dependent choices by Lévy [19] and the ordinal variants of Zorn's lemma [27] by Wolk.

\section{Acknowledgments}

We thank the communities of researchers who contributed to develop the material we built on, and in particular Camille Noûs, from the Cogitamus Lab, who embodies the collective and collaborative nature of scientific research.

The ideas in Section 4.3 derived from investigations led by Charlotte Barot [1]. The second author thanks Valentin Blot and Étienne Miquey for numerous fruitful discussions on the axiom of dependent choice and bar induction.

${ }^{8}$ We define the value of $\alpha$ as equations to remain agnostic on the representation of a function to $\mathbb{B}$, see 2.2 . 


\section{References}

[1] Charlotte Barot. Two [choice] principles involved in the intuitionistic completeness theorem. Master thesis, University Paris-Diderot, 2017.

[2] Josef Berger. The fan theorem and uniform continuity. In Proceedings of the First International Conference on Computability in Europe: New Computational Paradigms, CiE'05, page 18-22, Berlin, Heidelberg, 2005. SpringerVerlag.

[3] Josef Berger. A decomposition of Brouwer's fan theorem. J. Logic \& Analysis, 1, 2009.

[4] Josef Berger and Hajime Ishihara. Brouwer's fan theorem and unique existence in constructive analysis. Mathematical Logic Quarterly, 51(4):360-364, 2005.

[5] Josef Berger, Hajime Ishihara, and Peter Schuster. The Weak König Lemma, Brouwer's Fan Theorem, De Morgan's Law, and Dependent Choice. Reports on Mathematical Logic, 2012(Number 47), 2012.

[6] Ulrich Berger. A computational interpretation of open induction. In 19th IEEE Symposium on Logic in Computer Science (LICS 2004), 14-17 July 2004, Turku, Finland, Proceedings, page 326. IEEE Computer Society, 2004.

[7] Crystal Cheung. Brouwer's Fan Theorem: An Overview. Master thesis, Cornell University, 2015.

[8] Thierry Coquand. A note on the open induction principle. Chalmers University, 1997.

[9] Thierry Coquand. About brouwer's fan theorem. Revue Internationale de Philosophie, 230:483-489, 2004.

[10] Thierry Coquand and Henri Lombardi. A logical approach to abstract algebra. Mathematical Structures in Computer Science, 16(5):885-900, 2006. See also http://www.cse.chalmers.se/ coquand/formal.html.

[11] Christian Espíndola. Semantic completeness of first-order theories in constructive reverse mathematics. Notre Dame J. Formal Logic, 57(2):281-286, 2016.

[12] Jean-Yves Girard. Linear logic. Theor. Comput. Sci., 50:1-102, 1987.

[13] Leon Henkin. The completeness of the first-order functional calculus. J. Symb. Log., 14(3):159-166, 1949.

[14] Jean E. Rubin Herman Rubin. Equivalents of the Axiom of Choice. Elsevier, 1963.

[15] William A. Howard and Georg Kreisel. Transfinite induction and bar induction of types zero and one, and the role of continuity in intuitionistic analysis. J. Symb. Log., 31(3):325-358, 1966.

[16] Hajime Ishihara. Constructive reverse mathematics: Compactness properties. From Sets and Types to Topology and Analysis: Towards Practicable Foundations for Constructive Mathematics, 48, 102005.

[17] Hajime Ishihara. Weak König's Lemma Implies Brouwer's Fan Theorem: A Direct Proof. Notre Dame J. Formal Logic, 47(2):249-252, 042006.

[18] Thomas J. Jech. The Axiom of Choice. Dover Books on Mathematics Series. Courier corporation, 1973.

[19] Azriel Lévy. The interdependence of certain consequences of the axiom of choice. Fundamenta Mathematicae, 54(2):135-157, 1964.

[20] Jean-Claude Raoult. Proving open properties by induction. Information Processing Letters, 29(1):19-23, 1988.

[21] Davide Rinaldi and Peter Schuster. A universal Krull-Lindenbaum theorem. Journal of Pure and Applied Algebra, 220(9):3207 - 3232, 2016.

[22] Davide Rinaldi, Peter Schuster, and Daniel Wessel. Eliminating disjunctions by disjunction elimination. The Bulletin of Symbolic Logic, 23(2):181-200, 2017.

[23] Dana Scott. Completeness and axiomatizability. In Proceedings of the Tarski symposium, pages 411-435, 1974.

[24] Stephen G. Simpson. Subsystems of Second Order Arithmetic. Perspectives in Logic. Cambridge University Press, 2nd edition, 2009. 
[25] Richard E. Vesley Stephen Kleene. The foundations of intuitionistic mathematics. North-Holland, 1965.

[26] Wim Veldman. Brouwer's fan theorem as an axiom and as a contrast to kleene's alternative. Arch. Math. Log., 53(5-6):621-693, August 2014.

[27] Elliot S. Wolk. On the principle of dependent choices and some forms of Zorn's lemma. Canad. Math. Bull., 26(3), 1983. 